\title{
INVERSE TERNARY CONTINUED FRACTIONS
}

\author{
BY D. N. LEHMER
}

In Jacobi's extension of the continued fraction algorithm* we are concerned with three series of numbers given by the recursion formulas

$$
\begin{aligned}
& A_{n}=q_{n} A_{n-1}+p_{n} A_{n-2}+A_{n-3}, \\
& B_{n}=q_{n} B_{n-1}+p_{n} B_{n-2}+B_{n-3}, \\
& C_{n}=q_{n} C_{n-1}+p_{n} C_{n-2}+C_{n-3},
\end{aligned}
$$

with initial values $1,0,0$ for $A ; 0,1,0$ for $B$, and $0,0,1$ for $C$. We have called this series of numbers $\left(A_{n}, B_{n}, C_{n}\right)$ the convergent sets, and the series of numbers $\left(p_{n}, q_{n}\right)$ the partial quotient sets of a ternary continued fraction. $\dagger$ It is well known that if the partial quotient sets recur periodically the ratios $A_{n} / B_{n}, A_{n} / C_{n}$ and $B_{n} / C_{n}$ approach cubic irrationalities except in certain special cases where they approach quadratic irrationalities $\ddagger$ or where they approach no limit at all. The cubic irrationalities when they exist are connected by a linear fractional relation with the roots of the characteristic cubic

$$
\left|\begin{array}{ccc}
A_{k-2}-\rho & B_{k-2} & C_{k-2} \\
A_{k-1} & B_{k-1}-\rho & C_{k-1} \\
A_{k} & B_{k} & C_{k}-\rho
\end{array}\right|=0,
$$

connected with the purely periodic ternary continued fraction $\left(p_{1}, q_{1} ; p_{2}, q_{2} ; \cdots ; p_{k}, q_{k}\right)$ formed of those partial quotient pairs that recur. This characteristic cubic we write $\rho^{3}-M \rho^{2}+N \rho$ $-1=0$, where $M=A_{k-2}+B_{k-1}+C_{k}$, and

$N=A_{k-2} B_{k-1}-A_{k-1} B_{k-2}+B_{k-1} C_{k}-B_{k} C_{k-1}+C_{k} A_{k-2}-A_{k} C_{k-2}$.

We shall confine ourselves in what follows to purely periodic

* Jacobi, Werke, vol. VI, pp. 385-426.

$\dagger$ Proceedings of the National Academy of Sciences, vol. 4 (1918), p. 360.

$\ddagger \mathrm{J}$. B. Coleman, American Journal of Mathematics, vol. 52, No. 4, October, 1930 . 
fractions. Those fractions which have a finite number of nonperiodic partial quotient pairs to start with introduce no new difficulties. The irrationalities which they determine are simply related to the fraction obtained by ignoring the non-periodic pairs.

Two ternary continued fractions $\left(p_{1}, q_{1} ; p_{2}, q_{2} ; \cdots ; p_{n}, q_{n}\right)$ and $\left(p_{n}, q_{n} ; p_{n-1}, q_{n-1 n} ; \cdots ; p_{2}, q_{2} ; p_{1}, q_{1}\right)$ will be called inverse. If a purely periodic ternary continued fraction be set up with the first of these as its period and another with the second as its period the two continued fractions will not necessarily have the same discriminating cubic, unless the partial quotient pairs read backward and forward the same. Thus the cubic which corresponds to the fraction $(1,1 ; 3,2 ; 4,1)$ is $\rho^{3}-14 \rho^{2}-1=0$ and that corresponding to the fraction $(4,1 ; 3,2 ; 1,1)$ is $\rho^{3}-17 \rho^{2}$ $+3 \rho-1=0$. The irrationalities defined by these cubics are different. It is the purpose of this paper to show that if the $p$ 's and $q$ 's are given by the equations

$$
p=\alpha t+\beta, q=\gamma t+\delta, .
$$

where $\alpha, \beta, \gamma, \delta, t$ are any given integers whatever, positive, negative or zero, then the discriminating cubic is the same for the fraction and its inverse. This theorem we establish by induction, but in the treatment by this method it appears that it is necessary to drive along simultaneously a group of assumed relations. This peculiarity, seldom met with elsewhere, seems to be characteristic of the proofs by induction of theorems in continued fractions of higher orders.

Let the $n$th convergent set of the first fraction be $\left(A_{n}, B_{n}, C_{n}\right)$ and of the second be $\left(A_{n}^{\prime}, B_{n}^{\prime}, C_{n}^{\prime}\right)$, and let the coefficients of the discriminating cubic of the first be $M$ and $N$ and of the second $M^{\prime}$ and $N^{\prime}$. We have to show that if the equations (1) hold, then $M=M^{\prime}$ and $N=N^{\prime}$. These equations are easily verified, for $n=1,2, \cdots$. We assume them true for values of the index up to and including $n$. We consider then the fraction with $n+1$ partial quotient pairs: $\left(p_{1}, q_{1} ; p_{2}, q_{2} ; \cdots ; p_{n}, q_{n} ; p, q\right)$ where $p$ and $q$ also satisfy equations (1) and let the $n$th convergent set of the inverse of this fraction be $\left(A_{n}^{\prime \prime}, B_{n}^{\prime \prime}, C_{n}^{\prime \prime}\right)$. By the formula given on page 361 of my note on Jacobi's algorithm (loc. cit.), we have for the last three convergent sets of this fraction: 


$$
\begin{aligned}
& \left(C_{n-2}^{\prime}, p C_{n-2}^{\prime}+A_{n-2}^{\prime}, q C_{n-2}^{\prime}+B_{n-2}^{\prime}\right), \\
& \left(C_{n-1}^{\prime}, p C_{n-1}^{\prime}+A_{n-1}^{\prime}, q C_{n-1}^{\prime}+B_{n-1}^{\prime}\right), \\
& \left(C_{n}^{\prime}, \quad p C_{n}^{\prime}+A_{n}^{\prime}, \quad q C_{n}^{\prime}+B_{n}^{\prime}\right) .
\end{aligned}
$$

From the hypothesis that $M=M^{\prime}$ we have

$$
A_{n-2}+B_{n-1}+C_{n}=A_{n-2}^{\prime}+B_{n-1}^{\prime}+C_{n}^{\prime},
$$

and we wish to show that

$$
\begin{aligned}
A_{n-1}+B_{n}+q C_{n}+p C_{n-1}+C_{n-2}=A_{n-1}^{\prime \prime}+B_{n}^{\prime \prime}+C_{n+1}^{\prime \prime} \\
=C_{n-2}^{\prime}+p C_{n-1}^{\prime}+A_{n-1}^{\prime}+q C_{n}^{\prime}+B_{n}^{\prime} .
\end{aligned}
$$

Replacing $p$ and $q$ by their values from (1), we have

(3) $A_{n-1}+B_{n}+C_{n-2}+(\gamma t+\delta) C_{n}+(\alpha t+\beta) C_{n-1}$

$$
=A_{n-1}^{\prime}+B_{n}^{\prime}+C_{n-2}^{\prime}+(\gamma t+\delta) C_{n}^{\prime}+(\alpha t+\beta) C_{n-1}^{\prime} \text {. }
$$

If this equation is to hold for all values of $t$, we should have, equating coefficients of $t$,

$$
\gamma C_{n}+\alpha C_{n-1}=\gamma C_{n}^{\prime}+\alpha C_{n-1}^{\prime} .
$$

We now assume this equation to hold and proceed as before to $n+1$. In the resulting equation the coefficient of $t$ vanishes by virtue of equation (4). The terms independent of $t$ must satisfy the equation

(5) $\alpha C_{n}+\gamma\left(\delta C_{n}+\beta C_{n-1}+C_{n-2}\right)=\gamma \delta C_{n}^{\prime}+\alpha \delta C_{n-1}^{\prime}+\gamma B_{n}^{\prime}+\alpha B_{n-1}^{\prime}$.

This equation we also assume to be true for the values of $n$ in question, and on proceeding to $n+1$ we find the coefficients of $t$ vanish by virtue of (5). The remaining terms reduce by (4) and (5) to the equation

$$
\gamma C_{n-1}+\alpha C_{n-2}=\gamma A_{n}^{\prime}+\alpha A_{n-1}^{\prime} \text {. }
$$

On assuming this equation to hold and proceeding to the value $n+1$ we obtain equation (4) again. Equations (4), (5) and (6) thus form a closed system. We return to equation (3) and equate the parts independent of $t$. The resulting equation,

(7) $A_{n-1}+B_{n}+C_{n-2}+\delta C_{n}+\beta C_{n-1}$

$$
=A_{n-1}^{\prime}+B_{n}^{\prime}+C_{n-2}^{\prime}+\delta C_{n}^{\prime}+\beta C_{n-1}^{\prime},
$$


we also assume to hold for all values of the index up to and including $n$. Proceeding to $n+1$ we find that the coefficient of $t$ vanishes by virtue of (5), while the terms independent of $t$ yield the equation

(8) $A_{n}+\delta B_{n}+\beta B_{n-1}+B_{n-2}+C_{n-1}+\delta^{2} C_{n}+\beta \delta C_{n-1}+\delta C_{n-2}+\beta C_{n}$

$$
=A_{n}^{\prime}+\delta B_{n}^{\prime}+\beta B_{n-1}^{\prime}+B_{n-2}^{\prime}+C_{n-1}^{\prime}+\delta^{2} C_{n}^{\prime}+\beta \delta C_{n-1}^{\prime}+\delta C_{n-1}^{\prime}+\beta C_{n}^{\prime} \text {. }
$$

If we assume this equation and proceed to $n+1$, we find that the resulting equations are found to be true by virtue of the preceding equations. Equations (4), (5), (6), (7), and (8) with equation (2) form then a closed system of equations, all of which may be verified for $n=1,2,3, \cdots$. Assuming them true for values of the index up to $n$, they therefore hold for values up to $n+1$. They therefore hold in general.

The corresponding discussion for the assumed equation $N=N^{\prime}$ leads to the following set of equations, where for shortness we write $\left(A_{i}, B_{j}\right)$ for $A_{i} B_{j}-A_{j} B_{i}$ :

$$
\begin{gathered}
\left(A_{n-1}, B_{n}\right)+\left(A_{n-2}, C_{n}\right)+\left(B_{n-1}, C_{n}\right) \\
\quad=\left(A_{n-1}^{\prime}, B_{n}^{\prime}\right)+\left(A_{n-2}^{\prime}, C_{n}^{\prime}\right)+\left(B_{n-1}^{\prime}, C_{n}^{\prime}\right) . \\
\text { (10) } \gamma\left(A_{n-1}, C_{n}\right)+\alpha\left(B_{n}, C_{n-1}\right)=\gamma\left(A_{n-1}^{\prime}, C_{n}^{\prime}\right)+\alpha\left(B_{n}^{\prime}, C_{n-1}^{\prime}\right) . \\
\text { (11) } \gamma\left(A_{n}, C_{n-2}\right)+\alpha\left(C_{n}, B_{n-2}\right) \\
\quad=(\alpha \delta-\beta \gamma)\left(A_{n}^{\prime}, C_{n-1}^{\prime}\right)+\gamma\left(C_{n-1}^{\prime}, B_{n}^{\prime}\right)+\alpha\left(A_{n}^{\prime}, B_{n-1}^{\prime}\right) .
\end{gathered}
$$

(12) $\gamma\left(A_{n-2}, C_{n-1}\right)+\alpha\left(C_{n-2}, B_{n-1}\right)$

$$
\begin{aligned}
& =\gamma\left(A_{n}^{\prime}, B_{n-1}^{\prime}\right)+\alpha\left(A_{n-1}^{\prime}, C_{n}^{\prime}\right) . \\
& \left(A_{n-1}, B_{n}\right)+\left(A_{n-1}, C_{n-2}\right)+\left(B_{n}, C_{n-2}\right)+\delta\left(A_{n-1}, C_{n}\right) \\
& +\beta\left(B_{n}, C_{n-1}\right)=\left(A_{n-1}^{\prime}, B_{n}^{\prime}\right)+\left(A_{n-1}^{\prime}, C_{n-2}^{\prime}\right)+\left(B_{n}^{\prime}, C_{n-2}^{\prime}\right) \\
& \quad+\delta\left(A_{n-1}^{\prime}, C_{n}^{\prime}\right)+\beta\left(B_{n}^{\prime}, C_{n-1}^{\prime}\right) . \\
& \left(A_{n}, B_{n-2}\right)+\left(A_{n}, C_{n-1}\right)+\delta\left(B_{n}, C_{n-1}\right)+\left(B_{n-2}, C_{n-1}\right) \\
& \quad+\delta\left(A_{n}, C_{n-2}\right)+\beta\left(A_{n-1}, C_{n-2}\right)=\left(A_{n}^{\prime}, B_{n-2}^{\prime}\right)+\left(A_{n}^{\prime}, C_{n-1}^{\prime}\right) \\
& \quad+\delta\left(B_{n}^{\prime}, C_{n-1}^{\prime}\right)+\left(B_{n-2}^{\prime}, C_{n-1}^{\prime}\right)+\delta\left(A_{n}^{\prime}, C_{n-2}^{\prime}\right) \\
& \quad+\beta\left(A_{n-1}^{\prime}, C_{n-2}^{\prime}\right) .
\end{aligned}
$$

This is found to be a closed system of equations, and the assumption of any one is justified by the others. A simple trial verifies them for the values of $n=1,2,3$. They are then true in general. We have then the following theorem. 
THEOREM 1. In any periodic, ternary continued fraction the discriminating cubic is not changed when the order of the partial quotient pairs is inverted provided that the partial quotient pairs satisfy the equations

$$
p_{i}=\alpha t+\beta, q_{i}=\gamma t+\delta,
$$

where $\alpha, \beta, \gamma$ and $\delta$ are any fixed integers, positive, negative or zero, and the parameter $t$ also takes any integer values positive, negative or zero.

As an interesting special case we may take $\alpha$ or $\gamma$ equal to zero. Taking $\alpha=\delta=0, \gamma=1$, we get the following theorem.

Theorem 2. The discriminating cubic is not changed by inversion of order of the partial quotient sets in any periodic ternary continued fraction in which $p$ is fixed while $q$ takes any values, or. $q$ is fixed while $p$ takes any values.

Such continued fractions might be called linear, since the equation between $p$ and $q$ requires them to be the coordinates of points on a line. With some difficulty, due to the complicated algebra involved, we have shown that if in a quaternary continued fraction with periodic partial quotient sets $(p, q, r)$ we have the equations

$$
p_{i}=\alpha t+\beta, q_{i}=\gamma t+\delta, r_{i}^{\prime}=\lambda t+\mu,
$$

where $\alpha, \beta, \gamma, \delta, \lambda, \mu$ are fixed integers, positive, negative or zero, while the parameter $t$ takes any integer values, positive, negative or zero, then the order of the partial quotient sets may be inverted without changing the discriminating quartic. A similar theorem seems to hold for fractions of higher order, but a general proof is lacking.

The University of California 